\title{
THE LENR-CANR.ORG WEBSITE, ITS PAST AND FUTURE
}

\author{
J. ROTHWELL AND E. STORMS \\ LENR-CANR.org \\ 1954 Airport Road, Suite 204, Chamblee, GA 30341 \\ JedRothwell@mindspring.com
}

\begin{abstract}
The LENR-CANR.org web site has proven to be a popular source of information about cold fusion. This site has distributed more full text papers about LENR than any other source. In addition, it contains many features that allow easy search and insertion of the discovered references into a document.
\end{abstract}

People have recognized the need for a web site for cold fusion, but the form this should take was never clear enough to permit its creation. After ICCF-9, the two authors collaborated in creating the present format. A library of nearly 3000 references was available (ES) and this was made the basis for the site. In October the site was renamed LENR-CANR.

The web site content includes some introductions to the field, photographs of equipment, letters and opinions about cold fusion, full text copies of 210 technical papers, and a complete bibliography of papers having a relationship to the field. Most of the full-text papers appeared in ICCF conference proceedings or journals. A few are opinion pieces from magazines or historical reviews. Our selection is limited because we must get permission to reprint from the author or the publisher. Some cold fusion papers are available in permanent, free, online archives, such as the Japanese Journal of Applied Physics online edition ${ }^{1}$ and Wired Magazine. ${ }^{2}$ When these external web sites assign papers unique, permanent addresses, we upload a link into a Library pointing to the paper in the other web site, rather than uploading a copy. This saves space, and gives the reader a chance to explore these other sites. When papers are uploaded into transient sites we ask permission to copy them.

The Library also contains a bibliography of approximately 3000 papers that can be searched by author, title, journal or by using a few general keywords. The database is maintained on our computers with a bibliographic program call EndNote, which allows more sophisticated searches. It also allows references to be placed easily in a paper, numbered automatically and printed using any chosen format. This library will grow and will continue to be a unique source of information about LENR. To make this search easier using EndNote, a CD disc is available for purchase.

The original purpose of LENR-CANR was to make detailed technical information about cold fusion available to the general public. It succeeded beyond expecta- 


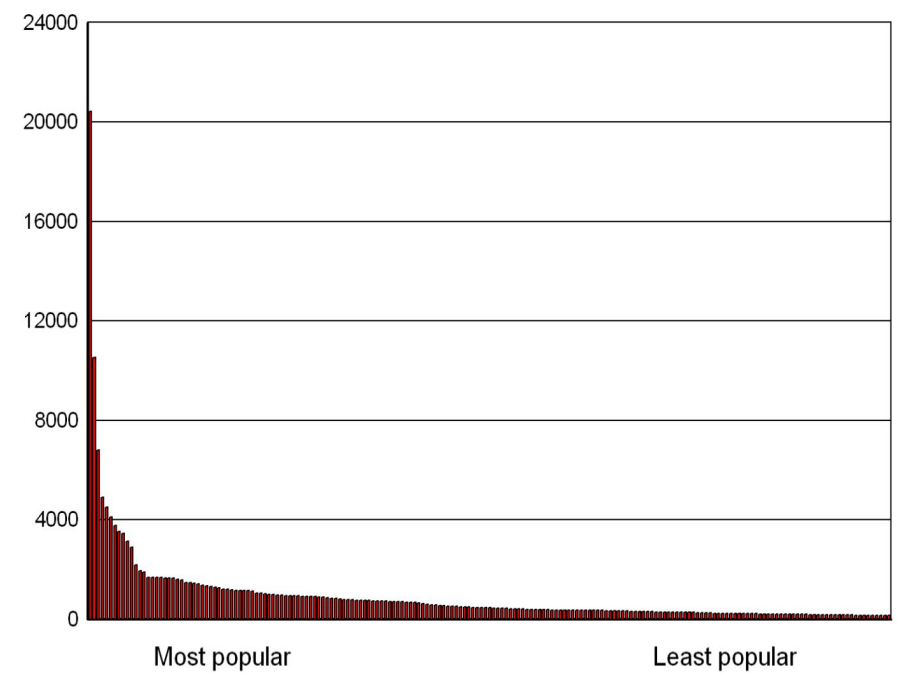

Figure 1. Distribution of hits per paper. There are approximately 3 hits per download.

tions. Readers have downloaded 71,000 copies of papers as of September 2003. This is probably more than the total number of papers distributed in proceedings, journals and other sources combined. This enthusiastic response indicates that many people are open-minded and seriously interested in the subject. The papers are not easy to read, and it is not likely that people are downloading them repeatedly merely to poke fun at them as some skeptics have done. It is not clear how many different people the site has attracted. The online web hosting utility program records precisely how many papers are downloaded daily, and how many visitors come each day, but it does not record how many people are visiting for the first time and how many are returning for a second or third visit. We do not ask visitors to sign in, so we have no way to record names or any other personal information, thus we cannot distinguish between unique visitors and repeat visitors. We estimate somewhere between 30,000 and 50,000 different visitors have come. The most popular papers on the site have been downloaded 3,000-6,000 times (see Fig. 1) Most others have been downloaded 100-600 times. Assuming that one person would not repeatedly download the same paper, and that some readers have not download the most popular papers, 30,000 is a reasonable minimum estimate. Some readers may download papers and then e-mail copies to their friends. It is not possible to keep track of how often this happens. Google and other search utilities have not found many copies of these papers uploaded at other web sites.

LENR-CANR has distributed 71,000 papers, or approximately 20 gigabytes of data. Operating the web site for 10 months has cost us $\$ 200$, or about 0.3 cents per paper. Distributing this many printed documents by mail would cost roughly $\$ 100,000$ in direct costs including postage, paper, copy machine toner and so on, and it would be a full-time job for several people. We distribute 300-600 papers 
per working day. Without the Internet, there is no way cold fusion researchers can reach a sympathetic audience. Thousands of people scattered around the world are interested in this subject and willing to read papers with an open mind. The potential audience even today may be in the hundreds of thousands. There is no cost-effective way to reach them except the Internet.

The skewed distribution of papers and the most popular titles tell us a great deal about the audience. Most people are interested in experiments, not theory. Papers describing the political aspects of cold fusion and the controversy are not very popular. The three most popular papers on the site are Passell's description of Kevin Wolf's radiation data at Texas A\&M, ${ }^{3}$ Mizuno's paper on plasma electrolysis, ${ }^{4}$ and Edmund Storms' Students Guide to Cold Fusion. ${ }^{5}$ Together they represent $21 \%$ of all download papers.

After the site was established a secondary purpose emerged: to preserve electronic manuscripts, data, photographs and other experimental evidence in an archive. Since, the field began 14 years ago a depressing amount of critical information has been lost. After Martin Fleischmann's secretary died, her computer disappeared along with many of Fleischmann's manuscripts in electronic form. In one of George Miley's papers ${ }^{6}$ the SEM photographs in Fig. 2(b) were preserved as high-definition images on a hard disk, but the images in Fig. 2(a) were lost and had to be scanned from a poor quality printed copy of the paper in a proceedings. Many other people, we have discovered, do not have their papers in electronic format, which makes distribution difficult. We urge all researchers to place as much of their critical information as possible on the site where it can be preserved and widely distributed.

Old documents can be converted from the paper format back into computer files, but the quality of the graphs and figures always degrades, and often equations cannot be recreated precisely as the author intended them to be. The process is time-consuming and error prone. When an author sends us a paper in electronic format, it usually takes us 15 min to register the paper in the Library index system, convert it into Acrobat format, and upload it. When an author provides a paper copy, it takes anywhere from four hours to a week to prepare it.

The site reached a peak of popularity just before summer vacation began at U.S. and European universities. After Memorial Day, readership fell abruptly from 4,000 papers per week to around 1,700. We hope this indicates that many readers are high school and college students, and there is some recent evidence for this: readership picked up during the summer session and at the beginning of the fall semester. On the other hand, this may indicate that nearly everyone in the world who wishes to read about cold fusion has already read the material on our site, and the market for cold fusion information is saturated. If authors would send us more papers readership might increase.

Papers presented at this conference will be available in full text on the site in a new section: ICCF10 Proceedings. In addition, a new journal focusing on the field is being created and submitted papers will also be available on line. ${ }^{7}$ As a result, 


\section{LENR-CANR Weekly Traffic}

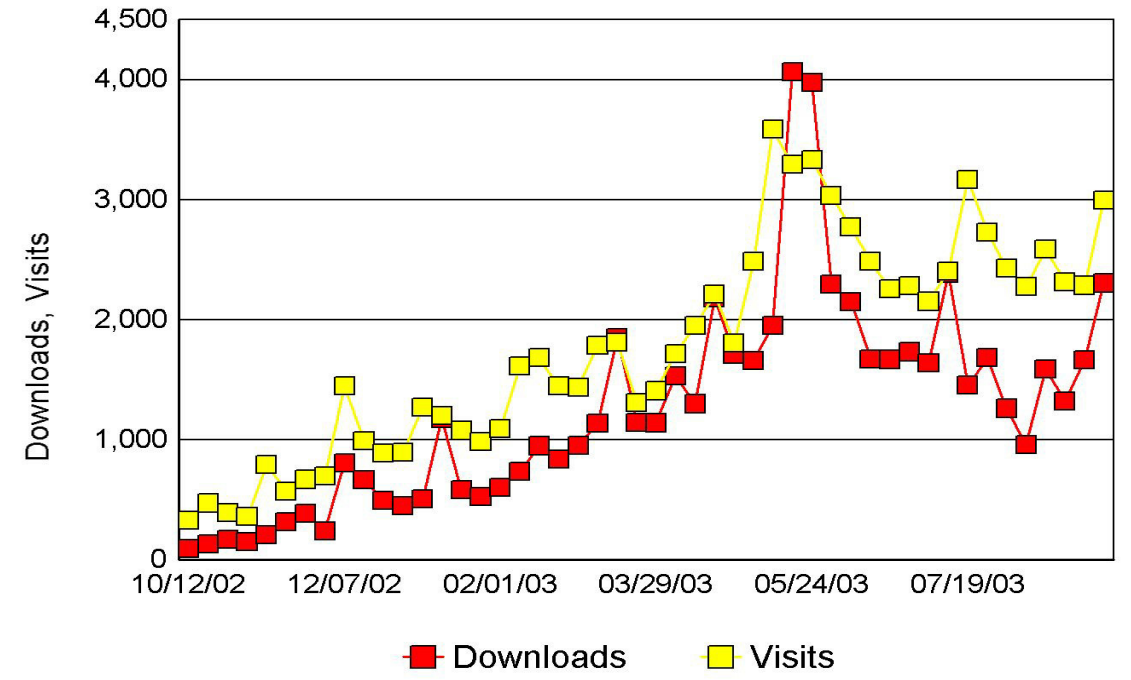

Figure 2. Number of downloads and visitors per week. We cannot tell how many visitors are returning and how many are new. For the latest version of this graph, see: http://www.lenrcanr.org/News.htm\#Downloads.

this web site is becoming a complete source of information about LENR and cold fusion.

\section{References}

1. http://jjap.ipap.jp/online/.

2. http://www.wired.com/.

3. T.O. Passell, Radiation Data Reported by Wolf at Texas A\&M as Transmitted by T. Passell, EPRI (1995).

4. T. Mizuno et al., in Confirmation of Heat Generation and Anomalous Element Caused by Plasma Electrolysis in the Liquid, Eigth International Conference on Cold Fusion, 2000 (Lerici (La Spezia), Italy: Italian Physical Society, Bologna, Italy).

5. E. Storms, A Student's Guide to Cold Fusion, LENR-CANR.org (2003).

6. G.H. Miley and J.A. Patterson, J. New Energy 1(3), 5 (1996).

7. P.L. Hagelstein, in On the New Electronic Journal, 10th International Conference on Cold Fusion, 24-29 August 2003, Cambridge, MA: LENR-CANR.org. 